\title{
The Design of Measurement Error Correction System for Ship Optical Theodolite
}

\author{
Yu Zou ${ }^{1, a}$ and Kai Li $i^{2, b}$ \\ ${ }^{1,2}$ China Satellite Maritime Measurement and Control Department, Jiangyin, China \\ a294244476@qq.com, b1650605@qq.com
}

Keywords: Ship Measuring Equipment; Optical theodolite; Error correction; System design

\begin{abstract}
The ship measurement device to perform the measurement task, influence by wind, tide and experience of ship hull navigation, drift motion, swing movement: the tracking measuring element will be the superposition of these rolling motion affect measurement accuracy. To eliminate the influence of the ship shaking data when carrying on the follow-up processing. In order to meet the needs of the flight test and control of the sea, it is necessary to make up for the limitation of the use of the fixed station and the mobile station of the vehicle by means of optical theodolite equipment loaded on the ship. In this paper, based on the practical engineering requirement, the measuring system composed of small ships in ship loading optical theodolite, the measurement data are developed ship swaying error model obtained can effectively eliminate the effects of processed ship data and ground-based optical theodolite data processing method, and designs a reasonable ship optical theodolite data process. The data processing of error correction, system error correction and target track calculation of optical measurement data are realized.
\end{abstract}

\section{Introduction}

Optical theodolite is the main tracking equipment of various types of flight test, which can obtain high precision angle measurement data. Compared with other methods such as radar and GPS, it has the advantages of high measurement accuracy, timely acquisition target, stable and reliable performance, and no interference from ground clutter. The measurement is intuitive and stable, and the film or CCD, the image can be used as the display of the command and control, and can also be used as the flight path. Because of its stable performance, it is often used to identify low and medium precision radio measurement equipment. The disadvantages of optical theodolite distance is limited and influenced by the weather with the aircraft flight test: the flight distance more and more far, navigation area is more and more big; and the progress of technology of electronic equipment, high precision measurement of radio measuring equipment has gradually become the main means of. However, due to the interference of ground objects in the low elevation angle of the initial section of the flight test, the influence of the state black zone is very important. In the initial flight test and reentry, radio measurement equipment are often unable to capture the target, "optical theodolite is not affected, to obtain high precision measurement data is reliable and stable, its irreplaceable role, is still the main measuring methods of various types of flight test of A. Optical theodolite and radio measurement equipment complement each other to complete the measurement of various types of flight test.

At present, the optical theodolite measuring equipment can be divided into many types, such as the ground load, ship borne airborne, satellite borne and so on. Large number of fixed equipment, a variety of types, usually for large equipment installed in a fixed instrument tower. The vehicle mounted transport equipment is arranged on the movable vehicle, and is arranged on the measuring ground before the flight test, and is installed on the ground which is prepared in advance or is temporarily installed on the ground. ship equipment is the optical equipment installed on large ships, in the ocean station, such as measuring ship. The basic equipment is simple calibration, the measurement of metadata interference is less, the station is fixed, so that the scope of measurement and control is limited to the fixed position. Because of the two-dimensional coordinate information of single theodolite can only get the space target, so in order to obtain three-dimensional 
coordinates of the target in space, at least more than two sets of optical theodolite is used to calculate the target position by way of intersection. In practical work, the problem of the error of the positioning of the flying target caused by the poor intersection geometry is often encountered. Vehicle or ship equipment can provide a more flexible layout scheme, to a greater extent improve the flight target localization accuracy with vehicle transportation equipment has a certain flexibility, but the relative aircraft flight distance, the maneuvering performance can not meet the requirement of control for. At the same time, China's limited land area, and various types of flight test flight distance increasing, flight zone expansion often across the sea and safety considerations based on the marine navigation area is more economical and reasonable, namely measuring ship measuring equipment - more can effectively meet the measurement and control of various types of flight test requirements.

\section{Shipboard Optical Theodolite Equipment}

Optical measurement using a remote non-contact way, only a part of the ground detection and reception equipment; simple, flexible, the need for external support conditions are less easy to row. Optical measurement has become an important means of early measurement of flight test. With the development of radar technology, radar measurement to replace the status and role of a considerable part of optical measurement equipment in the flight test, optical measurement equipment makes a considerable number of performance remains stable and reliable in sealed condition. In the initial stage of flight test, the optical measurement equipment is still irreplaceable under the condition of low elevation angle. The next flight test flight distance increased the safety and economic considerations, will build more traffic area in the ocean, the construction control network must consider these factors. Fixed optical theodolite equipment placed in the ship; cooperate with other related equipment to form ship optical theodolite measurement system, the system can comprehensively consider meteorological factors, sea route, based on the flexible layout in the observation area is more ideal. It is more suitable for the measurement and control of aerospace vehicles, and enhances the measurement capability of the system.

Therefore, the equipment of the ship borne optical theodolite is a powerful supplement to the measurement method of the early stage of the flight test. Research on ship borne equipment angle data attitude error correction method; will solve a key problem of ship borne optical theodolite measurement data processing, combined with terrestrial optical theodolite data processing method, design reasonable with the data of ship borne optical characteristics of the theodolite system management system can efficiently and accurately on the ship; load data processing measurement of optical theodolite. At the same time, it provides a reliable reference model for the analysis of other shipboard radio equipment and the measurement data of airborne airborne measurements in the future. The ship optical theodolite to station cross location geometric error is reduced when the optical theodolite measurement system, improve the overall control capacity of various types of flight test, and provide support for all types of flight test. To make up for the shortage of the land area of our country, which can not provide full coverage.

\section{Design of Data Processing System for Error Correction of Ship Optical Theodolite}

The basic condition and working characteristics of the shipboard equipment must be taken into account when dealing with the measured data of the shipboard equipment. The design of the data processing system must first establish the error correction model which accords with the real operating conditions. Then the specific error correction method is deduced. Finally, according to the characteristics of the business, targeted to solve the existing difficulties, to design a specific data processing process.

Error Correction Model of Ship Borne Optical Theodolite. The basic requirements of the mathematical model, simply is accurate, concise, correct, the relationship between the nature of non modeling problems so as not to be omitted, the model is too complex, this mainly for the correction of measurement error of small boats loaded optical theodolite, without considering the effects of 
hull deformation, at the same time that through debugging and calibration of equipment can eliminate the measurement equipment market level. The traditional ship data processing is to ship under the condition of measuring data correction to the inertial navigation platform center and then follow-up treatment, the method will ship under observation data is amended to the time of ship rocking, the ship will measure as a fixed site for further processing, can refer to the standard fixed station calibration and data processing mode. The system error elimination is more convenient, the following processing flow is clearer, the processing efficiency is higher.

The initial optical theodolite mainly uses film camera to record the flight target. Visible light is the main object of equipment measurement. And all the earth object infrared radiation, especially military targets, such as aircraft, missiles and other needs to consume energy, a part of energy will be converted into heat energy; the surface temperature, optical and infrared measurement using infrared thermal imaging technology can greatly expand the optical design effectively by distance, and optical theodolite life is long, with the transformation of infrared equipment can also dig optical equipment to maximize the use value. In order to improve the efficiency in the use of optical theodolite, a large number of optical theodolite have been transformed, the infrared measuring equipment installed in the original visible light measurement based on metadata will be measured only ship correction to not ship shaking, can directly use the existing data processing method.

The shipboard theodolite error correction model using small ships as carrier equipment without considering ship hull deformation, in order to facilitate the direct use of the existing fixed stand calibration parameters and equipment parameters; at the same time to consider the future of infrared data processing, ship equipment sloshing into standard fixed station $\mathrm{P}$ for further processing. In a moment, to measure the flight target by Hull shaking effect of shipboard theodolite, this coordinate system is affected by the ship ship motion measurement system: suppose the same time will eliminate the effect of ship rocking, the state of the measuring coordinate system called assumed coordinate system (the measurement measurement fixed site is equivalent to fixed on the sea measurement coordinate system), the ship borne equipment of ship motion coordinate measuring coordinates with the same goal of establishing relations in the assumed coordinate measuring coordinates. 2 sets of measuring system uses the same inertial coordinate system, the coordinate measuring system of the ship design target in inertial navigation system under the horizon (Xa, Ya, $\mathrm{Za}$ ), assumes that the measurement system under the target in inertial coordinate system of the earth $(\mathrm{Xc}, \mathrm{Yc}, \mathrm{Zc})$, the following relationship exists:

$$
\left[\begin{array}{c}
X a \\
Y a \\
Z a
\end{array}\right]=\left[\begin{array}{c}
X c \\
Y c \\
Z c
\end{array}\right]
$$

The coordinates of the target under the inertial navigation system (Xa, Ya, Za):

$$
\left[\begin{array}{l}
X a \\
Y a \\
Z a
\end{array}\right]=T^{-1}\left[\begin{array}{l}
X_{1} \\
Y_{1} \\
Z_{1}
\end{array}\right]+T\left[\begin{array}{l}
X_{0} \\
Y_{0} \\
Z_{0}
\end{array}\right]
$$

Among them, $(\mathrm{X} 1, \mathrm{Y} 1, \mathrm{Z} 1)$ is the coordinate of the target in the ship rolling measurement coordinate system (X0, Y0, Z0), which is the coordinate of the assumed station $\mathrm{O}$ in the inertial coordinate system. It can be obtained by ship coordinate and other ship borne equipment: $\mathrm{T}$ is the transition matrix of the coordinate system in the inertial navigation system. The coordinates of the target in the inertial navigation system $(\mathrm{XC}, \mathrm{YC}, \mathrm{ZC})$ are assumed:

$$
\left[\begin{array}{l}
X c \\
Y c \\
Z c
\end{array}\right]=\left[\begin{array}{l}
X \\
Y \\
Z
\end{array}\right]+\left[\begin{array}{l}
X_{0} \\
Y_{0} \\
Z_{0}
\end{array}\right]
$$

Among them $(\mathrm{X}, \mathrm{Y}, \mathrm{Z})$ is the coordinate of the object in the measurement coordinate system, and 
can be obtained by the repeated measurement. The formula (2), the formula (3) into the type (1), there is

$$
T^{-1}\left[\begin{array}{l}
X 1 \\
Y 1 \\
Z 1
\end{array}\right]+T\left[\begin{array}{l}
X_{0} \\
Y_{0} \\
Z_{0}
\end{array}\right]=\left[\begin{array}{l}
X \\
Y \\
Z
\end{array}\right]+\left[\begin{array}{l}
X_{0} \\
Y_{0} \\
Z_{0}
\end{array}\right]
$$

The formula (4) is the formula of the small and medium sized ship borne optical theodolite in the inertial navigation system, and the measurement of the target by the two measuring systems. A new error source for the subsequent survey vessel can be conveniently converted into a matrix into the equation.

The first record of the ship inertial navigation system according to the data, used to guide the transformation matrix $\mathrm{T}$ horizon coordinates affected by the data of ship rocking is derived; by Cartesian coordinate measuring system of ship rocking test element A1E1R1 calculation shake the measurement coordinate system in ship (X1, Y1, Z1); the ship body coordinate and the position data of the known solution must assume measuring coordinate origin in inertial coordinate horizontal coordinates (X0, Y0, Z0), affected by the data of ship sway calculation, inertial coordinate system of the ship coordinate system origin: because the ship swaying measurement system and measurement system of measurement is assumed the same target, the 2 coordinate system to the object coordinate inertial navigation coordinate system is the same as that of equation (1), the equations are assumed to measure the sit target coordinates $(\mathrm{X}, \mathrm{Y}, \mathrm{Z})$ under the target system can be obtained by means of inverse calculation.

Optical Theodolite after Data Processing Flow. The trajectory is the trajectory of the target state estimated from the measurement set from the same target. One of the final results of the data processing system of optical theodolite is the track. After many years of practical application, the data processing method has been very mature. The first device to record film or a CCD image data, to ensure no interference image information, through digital image processing to improve the visual quality of the image, such as the removal of the "noise" in the image, change the image brightness, color, enhance the image of some of the ingredients. It can restrain some components and transform the image to the image, so as to improve the quality of the image. Next, the position of the target in the image is obtained by the film or CCD image interpretation. Combined with the information of the image array, the azimuth angle and elevation angle of the target relative to the station are obtained. In the above work, image processing is a cross discipline which involves many research fields. This paper mainly discusses the data processing after obtaining relevant data.

To obtain relevant data after measurement, optical theodolite data processing and the basic process of the main work: (1) the preparation of basic data, basic data proofreading and revision, including the site and site data corresponding to the azimuth mark theory value; equipment related parameters, including the precision of equipment parameters, not parallel, infrared infrared moving base the geometric relations of parameters; comprehensive information, including zero time, origin and weather. (2) observation data preprocessing: measurement data, including visible and infrared observation data storage, the data need infrared optical axis of the measurement data storage; measuring data inspection and tracking analysis: time to check and statistics of the observation data, greatly outliers, identify, delete, replace the data breakpoint data interpolation; the measuring system error; metadata: the theoretical value of three standard range correction, complete system error correction, visible optical element infrared measurement system error correction and dynamic reference point operation link, eliminate the measuring equipment system error, calculation of infrared moving reference point for trajectory calculation using. (3) flight trajectory calculation: the atmospheric refraction correction is performed on the measured element; the influence of atmospheric refraction is eliminated; the multi station rendezvous includes visible light intersection, infrared intersection and multi type element intersection. (4) the accuracy of the statistical element and flight target track intersection: the statistical accuracy of the unit of measurement, the statistical accuracy of the flight target track. Compared with other data sources such as GPS and radar measurements. For example, the comparison between the high precision GPS counter and the 
measured element can accurately identify the accuracy of the measurement and control equipment, and provide a reliable basis for the development of the measurement and control scheme of the flight target.

The site optical theodolite data processing system to meet the needs of the foundation treatment, optical theodolite fixed vehicle equipment requirements, in such conditions, the site equipment is fixed on the base, was kept constant during the measurement data and measurement data recorded in the database, data analysis and inspection. The basic data in the range of standard theoretical value calculation of correction and measurement error correction system measuring system error element, based on the meteorological data of atmospheric refraction complete pitch angle, multi station intersection calculation of flight target track, the measurement accuracy and flight track statistics.

The system is able to measure the data of ground-based optical theodolite fixed station for accurate data processing, to complete the original entry, measuring element and basic data measuring data and statistics, check the quality of observation data analysis, system error correction, reference point calculation, measurement of atmospheric fold She Xiuzheng, multi station positioning trajectory calculation, important data recording and output etc..

\section{Conclusion}

This article is based on the demand of long distance flight target tracking measurement, the small and medium-sized ships and optical theodolite is built with onboard measurement system. The error sources and classification of ship borne measuring equipment are determined by studying the error characteristics of ship rocking data recorded by ins. Based on the ship rocking correction method of large space measurement ship, a basic model is established which is suitable for the measurement error correction of small and medium sized ship borne optical theodolites. Based on the data processing system of the optical theodolite of land-based fixed station, combined with the characteristics of ship borne measurement, the ship rocking correction and data processing flow of the ship optical theodolite equipment are designed. The conclusions are as follows: (1) measuring operation measurement ship on the sea, the ship rocked on the angle measuring element influence, if modeling unreasonable, the system error is difficult to eliminate, unable to meet the high precision measurement of optical solution requirements; (2) the correction method proposed in this paper can realize the measurement of effective correction the element of the visible light angle, and more adapt to the current data of infrared optical theodolite widely used an infrared equipment transformation mode of processing; (3) the flight target distance of ship borne optical theodolite is close, azimuth and pitching angle data by boat and greater data; and when the target is far away from the ship optical theodolite when the ship data element influence on angle becomes smaller; (4) near the route shortcut, residual azimuth angle and pitch angle than the stationary section, should use the ship maneuvering performance measurement high, comprehensive Considering the reasonable design of the station position, in order to improve the measurement accuracy. In a variety of measurement conditions can be very good to meet the requirements, this method has better effect on increasing the error correction, tracking data processing methods and improve the accuracy of exterior ballistic parameters are useful supplement. At the same time, the design based on the actual engineering verification program, can be used as a reference for future airborne other base station measurement method validation.

\section{References}

[1] R.Yuan, Q.Qiu. Photonics and Optoelectronics [M] Beijing: China Machine Press, 2014.(In Chinese)

[2] Y. BAR-SHALOM, T. E. FORTMANN. Tracking and Data Association [M]. Salt Lake City: Academic Press, 1988.

[3] S.S.BLACKMAN,R. POPOLI. Design and Analysis of Modern Tracking Systems [M]. Boston, London: Artech House, 1999. 
[4] C.H.Yu, Y.C.Pang, T.Yu. Visual c++Digital image processing and engineering application [M]: Beijing: China Railway Publishing House, 2012.(In Chinese)

[51 KLEUSBERG A. Comparing GPS and GLONASS[J]. GPS world. 1990(6): 52-54.

[6] M.Chai, H.Yu,W.H. Song,etc. Positioning method of optical radio measurement information fusion [J]. Acta optica Sinica, 2012,(32):158-164.(In Chinese)

[7] Z.Q.Ma,F.S. Guo,L. Y.Chen. Range geodesy [M] Beijing: National Defence Industry Press, 2004.(In Chinese)

[8] S.Y. Wang. Calibration method of azimuth reference for ship photoelectric theodolite [J] Journal of Changchun University of Science and Technology, 2014; 37(3): 13-16. (In Chinese)

[9] H.Liu, J.Han. MATLAB R2014a completely self-study a pass [M]. Beijing: Publishing House of electronics industry, 2015.(In Chinese)

[10]Y.H.Wang. Modern electrical control and PLC application technology [M]. Beijing: Beihang University Press, 2013.(In Chinese) 